San Jose State University

SJSU ScholarWorks

Master's Projects

Master's Theses and Graduate Research

2007

\title{
Morbidity and Mortality ofVery Low Birth Weight Infant Graduates of a Level Three Neonatal Intensive Care Unit
}

Maria Cortes

San Jose State University

Follow this and additional works at: https://scholarworks.sjsu.edu/etd_projects

Part of the Maternal, Child Health and Neonatal Nursing Commons

\section{Recommended Citation}

Cortes, Maria, "Morbidity and Mortality ofVery Low Birth Weight Infant Graduates of a Level Three Neonatal Intensive Care Unit" (2007). Master's Projects. 754.

DOI: https://doi.org/10.31979/etd.kc28-xbtn

https://scholarworks.sjsu.edu/etd_projects/754

This Master's Project is brought to you for free and open access by the Master's Theses and Graduate Research at SJSU ScholarWorks. It has been accepted for inclusion in Master's Projects by an authorized administrator of SJSU ScholarWorks. For more information, please contact scholarworks@sjsu.edu. 


\section{SAN JOSE STATE UNIVERSITY SCHOOL OF NURSING}

\section{MASTER'S PROGRAM PROJECT OPTION (PLAN B)}

\section{PROJECT SIGNATURE FORM}

STUDENT NAME

Maria E. Cortes

SEMESTER ENROLLED Spring '07

TITLE OF PROJECT

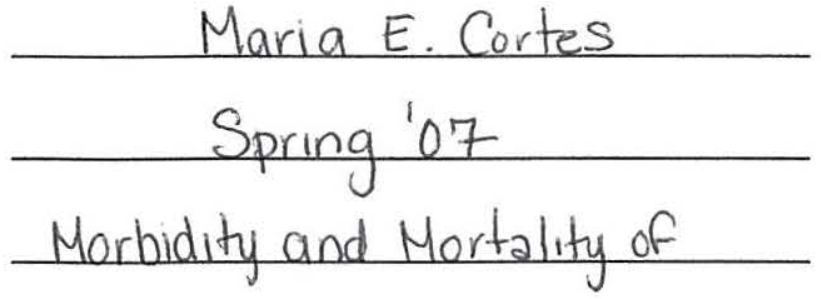
Very low Burth Weight Graduates of a Level Three Neonatal Intensive Care Unit.

\section{NAME OF JOURNAL}

Neonatal Network

(R)

The project and the manuscript have been successfully completed and meet the standards of the School of Nursing University. The project demonstrates the application of professional knowledge, clinical expertise, and scholarly thinking. An abstract of the project and two copies of the manuscript are attached.

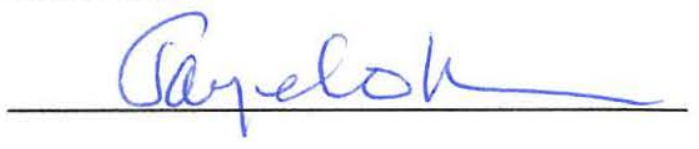

ADVISOR'S SIGNATURE

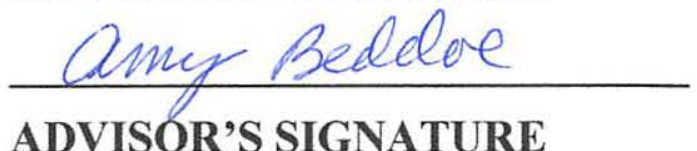

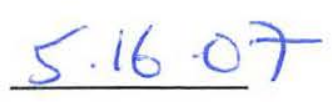

DATE

$5-16-07$

DATE

Please submit the form to the Graduate Coordinator. Attach abstract, two copies of the manuscript, and the documentation of submission to the journal (i.e., postal receipt). 
Morbidity and Mortality of Very Low Birth Weight Infant Graduates of a Level Three Neonatal Intensive Care Unit

Maria E. Cortes, RN, MS, FNP

\begin{abstract}
Purpose: To describe the morbidity and mortality of very low birth weight (VLBW) infant graduates of a level three neonatal intensive care unit (NICU) in a medically underserved population.
\end{abstract}

Design: A retrospective chart analysis of 181 live born infants at a regional tertiary center between 2004-2006.

Sample: Infants born with a birth weight of 425-1489 grams and gestational age of 23-40 weeks $(n=127)$.

Main outcome variable: Descriptive statistics were used to describe the incidence of associated VLBW morbidities presenting after discharge along with hospital readmissions and mortality rates.

Results: VLBW infants discharged from the NICU had chronic lung disease (24.4\%), seizures (3\%), hearing loss $(5.5 \%)$, cerebral palsy $(1.6 \%)$, mental retardation $(0.8 \%)$, and visual loss (0.8\%). They also showed abnormal neurodevelopmental findings (59\%), speech/language disorders (12.5\%) and behavioral/learning disorders (1.5\%). One death $(<1 \%)$ was reported after discharge. Forty-five children (35\%) were readmitted to the hospital after discharge accounting for 78 hospital admissions. 
Running head: MORBIDITY AND MORTALITY OF VERY LOW BIRTH WEIGHT INFANT GRADUATES

Morbidity and Mortality of Very Low Birth Weight Infant

Graduates of a Level Three Neonatal Intensive Care Unit

Maria E. Cortes, RN, MS, FNP

Jayne Cohen, DNSc, RNC

Amy Beddoe, $\mathrm{PhDc}, \mathrm{RN}$

San Jose State University

School of Nursing 


\begin{abstract}
Purpose: To describe the morbidity and mortality of very low birth weight (VLBW) infant graduates of a level three neonatal intensive care unit (NICU) in a medically underserved population.

Design: A retrospective chart analysis of 181 live born infants at a regional tertiary center between 2004-2006.

Sample: Infants born with a birth weight of 425-1489 grams and gestational age of 23-40 weeks $(\mathrm{n}=127)$.

Main outcome variable: Descriptive statistics were used to describe the incidence of associated VLBW morbidities presenting after discharge along with hospital readmissions and mortality rates.

Results: VLBW infants discharged from the NICU had chronic lung disease (24.4\%), hearing loss $(5.5 \%)$, seizures (3\%), cerebral palsy (1.6\%), mental retardation $(0.8 \%)$, and visual loss $(0.8 \%)$. They also showed abnormal neurodevelopmental findings $(59 \%)$, speech/language disorders $(12.5 \%)$ and behavioral/learning disorders $(1.5 \%)$. One death $(<1 \%)$ was reported after discharge. Forty-five children (35\%) were readmitted to the hospital after discharge accounting for 78 hospital admissions.
\end{abstract}


The number of very low birth weight infants (VLBW; birth weight less than 1500 grams) discharged home for follow-up in primary care settings has increased over the past several years due to rapid advances in the field of neonatology. The post-surfactant, post-steroid era of neonatology has led to a much greater survival rate of VLBW infants than previously expected. These high risk infants have a greater chance of developing illnesses with co-morbidities than do infants of normal birth weight. The Agency for Healthcare Research and Quality (2002) published a study in which VLBW infants, although accounting for only a small proportion of the total number of births in the United States, account for the highest mortality rate among neonates and the greatest number of morbidities among all newborns. Morbidities identified in the VLBW population included chronic lung disease (CLD), cerebral palsy, mental retardation, disorders of speech and language, behavior and learning disabilities, blindness and growth retardation (AHRQ, 2002).

\section{Literature Review}

Castro et al. (2004) reinforced earlier studies demonstrating an increased risk of developing cerebral palsy in children diagnosed with CLD. Bronchopulmonary dysplasia (BPD) is the most common form of CLD in premature infants and occurs as a result of prolonged mechanical ventilation. Short et al. (2003) demonstrated that infants with BPD had long-term adverse effects on cognitive and academic achievements. BPD has also been identified as a significant cause of prolonged hospitalization among VLBW infants (Klinger, Sirota, Lusky, \& Reichman, 2006). Additionally VLBW infants have been found more likely to suffer language deficits than infants of normal birth weight (Jansson-Verkasalo et al., 2004). Behavioral problems are also of concern in high risk infants. For instance, Reijneveld et al. (2006) reported 
that VLBW children are more likely to have behavioral and emotional problems detrimental for academic functioning at the age of five.

Hearing loss among VLBW infants was reviewed by Ari-Even Roth et al. (2006) who conducted a retrospective study to examine its prevalence. The study showed a low incidence of sensory-neural hearing loss and a relatively high incidence of conductive hearing loss.

Bronchopulmonary dysplasia was listed as one of the most significant factors for predicting the occurrence of conductive hearing loss. The National Center for Health Statistics reported 4.14 million births in the year 2005, in which $1.5 \%$ were reported as VLBW (Hamilton et al., 2007). The risk of early death for infants born at a VLBW is more than 100 times that of infants born at 2500 grams or greater. In 2003, nearly one-half of all infant deaths in the United States occurred in infants with a birthweight less than 1,000 grams (Mathews \& MacDorman, 2006). Medical and developmental outcomes of these VLBW infants are becoming increasingly important due to the continuing advancement of neonatal medicine and potential ethical concerns. This information is crucial for use in counseling families and potentially altering perinatal and neonatal care (Castro et al., 2004).

\section{Purpose}

The purpose of this research study was to describe mortality rates and morbidities affecting VLBW infants who were discharged from a level three neonatal intensive care unit (NICU) providing services to a medically underserved population. The specific focus of the study was on incidence of CLD, cerebral palsy, mental retardation, non-febrile seizures, visual, and hearing loss, as well as abnormal neurodevelopmental findings, speech/language disorders, behavioral/learning disorders, hospital admissions, and death after discharge. The study also 
assessed the importance of reporting follow-up assessments, interventions and outcomes on this vulnerable population.

\section{Methodology}

Setting

This study was conducted among patients discharged from a regional level three NICU in Northern California. The facility includes a tertiary level 40-bed NICU serving a population of approximately 650 newborns per year. The center qualifies as a medically underserved area facility (i.e., based on a shortage of primary care providers, geographical and demographic factors) and is one of only 23 regional centers in California. The study was approved by both the hospital and university institutional review boards.

\section{Design}

Patient data was obtained by retrospective review of medical record transcriptions and coding sheets. Data abstracted from these records included demographic information and six morbidities commonly associated with VLBW infants. These were: (a) CLD, defined as receiving supplemental oxygen at 36 weeks postmenstrual age; (b) cerebral palsy; (c) mental retardation; (d) non-febrile seizures; (e) visual loss; and (f) hearing loss (sensorineural and conductive). Additionally, data abstracted included abnormal neurodevelopmental findings, speech/language disorders, behavioral/learning disorders, hospital admissions after discharge, and information on death after discharge. Neurodevelopmental results were obtained through qualitative reports from high risk infant follow up visits and appropriately referred specialty care. Abnormal neurodevelopment was defined as presence of hypotonia, hypertonia, asymmetry, abnormal gait, gross motor delay, developmental delay, spasticity, and/or choreoathetoid movements. Qualitative reports were guided by the Amiel-Tison neurologic assessment (1987) 
which evaluates active and passive tone, strength, reflexes, joint angles, and posture. Deviations in any of these areas were noted as abnormal. A chart audit tool was used to obtain aggregate data. Infants were then categorized based on their chronological age at last documented chart entry in six categories: (a) 0-6 months; (b) 6.1-12 months; (c) 12.1-18 months; (d) 18.1-24 months; (e) 24.1-30 months; and (f) greater than 30 months. Descriptive statistics were used to portray the data in frequencies and percentages.

Sample

The target population included 181 VLBW infants admitted to the NICU were born during January 1, 2004 through September 30, 2006. Thirty seven of the VLBW infants expired prior to discharge yielding a mortality rate of $20.44 \%$. Seventeen infants were lost to follow up upon discharge from the NICU. A final sample of 127 children were included in this study. Birth weights ranged from 425-1489 grams and gestational age ranged from 23-40 weeks postmenstrual age. Sixty two of the survivors were male (49\%) and 65 were female (51\%). The majority of the survivors were Hispanic (69.3\%), followed by Black (7\%), White (6\%), Asian (2.4\%), Vietnamese (1.6\%), Arab (<1\%), and Filipino (<1\%). Racial information was listed as unknown or other in $12 \%$ of the records (see Table 1). Demographic information was comparable between infants who expired prior to discharge and those who survived after discharge. Of 127 children, only $10(8 \%)$ had private insurance, the remaining $110(92 \%)$ received public health care funding.

\section{Results}

Very low birth weight infants discharged from the NICU were diagnosed with CLD (24.4\%), hearing loss (5.5\%), cerebral palsy (1.6\%), non-febrile seizures (3\%), mental retardation (0.8\%), and visual loss (0.8\%) (see Table 2). Eight percent of children with CLD had 
ongoing oxygen requirements at the time of their last documented clinical visit $(n=4)$. Abnormal neurodevelopmental findings were found in $59 \%$ of the infants. Speech/language disorders were found among $12.5 \%$ of infants and behavioral/learning disorders were found among $1.5 \%$ (see Table 3). Forty-five children (35\%) were readmitted to the hospital after discharge accounting for 78 hospital admissions (see Table 4). Prior to discharge mortality was reported at $20.44 \%$. After discharge, mortality rate decreased to less than one percent. Three children demonstrated symptoms of cerebral palsy and were yet to be diagnosed at the time of the study indicating the incidence of cerebral palsy may be as high as four percent.

\section{Mental retardation}

Mental retardation accounted for one child (0.8\%) with Marfan syndrome born at 28 weeks gestational age with a grade 1 intraventricular hemorrhage. Cerebral palsy

Cerebral palsy was diagnosed in 2 children (1.6\%), one of which was born at 26 weeks gestational age and was diagnosed with CLD and grade two intraventricular hemorrhage prior to discharge. This child was later diagnosed with periventricular leukomalacia upon follow up with a neurologist. The other case of cerebral palsy was diagnosed in an infant born at 31 weeks gestational age with a grade one intraventricular hemorrhage at discharge and no CLD. Loss of vision

Visual loss was reported in one infant who was 33 weeks gestational age $(0.8 \%)$. Non-febrile seizures

Non-febrile seizures were reported among $3 \%$ of infants $(n=4)$. One child was born at 30 weeks gestational age with a cleft palate and birth weight of 1345 grams who had a normal head ultrasound at discharge from the NICU. This infant was readmitted shortly after discharge and 
was found to have a cerebral infarct with cerebral edema and associating seizures. The second case was born at 33 weeks gestational age of 1285 gram birth weight with possible Williams syndrome. The ultrasound of this child was normal at the time of discharge. The third child was born at 26 weeks gestational age with a birth weight of 1075 grams. The infant was born with congenital hydrocephalus, was diagnosed with a grade three intraventricular hemorrhage along with possible periventricular leukomalacia suggested by head ultrasound at time of discharge, and remains shunt dependent. The fourth child was born at 28 weeks gestational age with Marfan syndrome and microcephaly. The infant had a grade one intraventricular hemorrhage diagnosed prior to discharge from the NICU.

\section{Hospital readmissions}

Five children had four or more hospital readmissions (4\%). One of these children was born at 27 weeks gestational age with a birth weight of 530 grams who had four hospital admissions after discharge. The causes for these admissions included urinary tract infection, fever, respiratory distress, and lack of normal development. The next infant with multiple hospitalizations was the above mentioned infant born with Marfan syndrome (described above). This infant was hospitalized four times after discharge in which three were due to respiratory issues and one was for convulsions. An infant born at 35 weeks gestational age with multiple congenital anomalies and Goltz syndrome was readmitted five times. A term child with Cornelia de Lange's syndrome was admitted four times after discharge. The child with six hospital admissions was a term infant with a birth weight of 1250 grams with pulmonary agenesis. All admissions for this child were respiratory in nature. 


\section{Limitations}

One limitation of this study is the inclusion of a range of infant ages. Data such as school performance, behavioral problems and learning disabilities are typically diagnosed after one year of age and since a large portion of the sample was below this age, the percentiles presented may be underestimated. Further follow up of the same sample would clarify any missed cases not reported in this study. The study also fails to provide a control group for comparison, which limits the significance of the reported outcomes. The use of a single facility also presents a limitation since a multi-facility study may show different outcomes. Variances in medical and nursing care exist among facilities not only in the NICU, but in high risk infant follow up programs, and across different socioeconomic populations.

Qualitative neurological data was used to determine abnormal neurodevelopmental findings. Neurological data that is quantitative may serve as a better indicator of neurodevelopmental deficits, allowing for the grading and categorization of children based on the severity of their deficit. The descriptive study design did not provide explanations for patient morbidities or mortalities, nor for factors associated with risk of morbidity such as surfactant administration or prenatal administration of corticosteroids. Categorization of infants based on birth weight, gestational age, or grade of intracranial hemorrhage may have provided a different perspective as to the risk of neurodevelopmental impairment in this VLBW infant population.

\section{Discussion}

This research study reported current data on mortality rates and morbidities affecting VLBW infants discharged from a level three NICU that provided services to a medically underserved population. The morbidities of interest were CLD, cerebral palsy, mental retardation, non febrile-seizures, visual loss, hearing loss, abnormal neurodevelopmental 
findings, speech/language disorders, behavioral/learning disorders, and hospital admissions after discharge. Although data is lacking to compare the study group with another group, the results of this study appear to support the previous findings that indicate a high incidence of neonatal morbidity despite a decrease in VLBW infant mortality (Wilson-Costello et al., 2004). The frequency of hospital admissions after discharge demonstrated in this study positively correlated to Hintz et al.'s (2004) suggestion that improved survival of VLBW infants has led a greater risk for in-hospital morbidities for surviving infants.

Following discharge from the NICU, VLBW infants and their families continue to face a challenging time of uncertainty (Lasby, Newton, \& Platen, 2004). Ongoing medical concerns and complications frequently plague this vulnerable population and improvements in VLBW infant mortality does not represent improved neonatal morbidity. The future of VLBW infants is and has always been in the hands of the people who have cared for the infant, either in-utero, at birth, upon admission to the NICU, or after discharge from the NICU. The importance of preventing premature births is by far the most significant factor in the prevention of neonatal morbidity and mortality. Unfortunately, previous efforts in the prevention of premature births have not had a major effect in reducing the overall rate of prematurity (Hamilton et al., 2007). Therefore efforts must be made to ensure a positive outcome for these children after birth has occurred. It is vital for acute care nurses, physicians and other inpatient providers to use their knowledge of VLBW infants to create evidence based guidelines designed to improve the overall outcome of these tiny babies. In the community, public health nurses, high-risk infant follow up team members and primary healthcare providers including advanced practice nurses can best intervene through prompt identification of developing morbidities. The goal is for early intervention and rehabilitation in order to limit the extent of such morbidities and improve the quality of life for 
VLBW infant survivors. Evidence suggests that the extent of morbidities can be reduced if interventions are implemented early on. The outcome of an acute illness or injury depends not only on appropriate medical and surgical care but also on the recognition of a patients' need for comprehensive follow up care (Stucki, Stier-Jarmer, Grill, \& Melvin, 2005). Therefore an effort must be made to collaborate with parents and guardians of surviving infants regarding the importance of ongoing follow up visits for early identification and intervention of neonatal morbidities. A multi-disciplinary collaborative effort is vital for the best overall outcome of these children. There is a tremendous need for research among the disciplines in order to improve the outcome for future VLBW infants.

In summary, technology in medicine has excelled in the field of neonatology. With this advancement comes a decrease in neonatal mortality and an increase in survival of premature infants. This improvement in modern medicine contributes to an increasing number of high risk infants surviving after discharge and presenting themselves for care in primary care settings. Unfortunately, the increase in survival rates of VLBW infants has not decreased the incidence of morbidity. Very low birth weight infants are still at risk for long term morbidities such as CLD, cerebral palsy, mental retardation, and loss of vision and hearing. Long term follow-up of this population is necessary for a more accurate description of risk assessment and for improving quality of life.

Improvement of neonatal outcomes calls for further research aimed towards the prevention of preterm birth and the reduction of neonatal morbidity. Further controlled, large multicenter studies are needed to investigate the outcomes of VLBW infants and effectiveness of current evidence based practice guidelines. There is also a need for research dedicated to reducing the rate of hospital readmissions among infants discharged from the NICU. Neonatal 
nurses and inpatient providers are needed to research further evidence based practice guidelines aimed to optimize the health and outcome of the VLBW infant population.

\section{Acknowledgements}

This work was supported in part by Professional Nurse Traineeship Grant number 5NU00432-10 funded by the U.S. Department of Health and Human Services. We are extremely grateful to Balaji Govindaswami, MD, MPH, Christina Anderson, MD, Linda Taylor, RN, BSN, and May Loo, MD, FAAP for all their help and support in conducting this research. 


\section{References}

Agency for Healthcare Research and Quality. (2002). Evidence report/Technology assessment No. 70: Criteria for determining disability in infants and children: Low birth weight. Retrieved October 21, 2006 from www.ahrq.gov/clinic/epcsums/lbwdissum.htm

Amiel-Tison, C. (1987). Neuromotor status. In H. W. Taeusch \& M. W. Hogman (Eds.). Followup management of the high-risk infant. (pp. 115-126). Boston, MA: Little, Brown \& Company.

Ari-Even Roth, D., Hildesheimer, M., Maayan-Metzger, A., Muchnik, C., Hamburger, A., Mazkeret, R., et al. (2006). Low prevalence of hearing impairment among very low birthweight infants as detected by universal neonatal hearing screening. Archives of Disease in Childhood; Fetal Neonatal Edition, 91(4), F257-F262.

Castro, L., Yolten, K., Haberman, B., Roberto, N., Hansen, N., Ambalavanan, N., et al. (2004). Bias in reported neurodevelopmental outcomes among extremely low birth weight survivors. Pediatrics, $114,404-410$.

Hamilton, B. E., Minino, A. M., Martin, J. A., Kochanek, K. D., Strobino, D. M., \& Guyer, B. (2007) Annual summary of vital statistics. Pediatrics, 119, 345-360.

Hintz, S. R., Poole, W. K., Wright, L. L., Fanaroff, A. A., Kendrick, D. E., Laptook, A. R., et al. (2004). Changes in mortality and morbidities among infants born at less than 25 weeks during the post-surfactant era. Archives of Disease in Childhood; Fetal Neonatal Edition, 90, F128-F133.

Klinger, G., Sirota, L., Lusky, A., \& Reichman, B. (2006). Bronchopulmonary dysplasia in very low birth weight infants is associated with prolonged hospital stay. Journal of Perinatology, 26,640-644. 
Lasby, K., Newton, S., \& Von Platen, A. (2004). Neonatal transitional care. Canadian Nurse, 100(8), 18-23.

Mathews, T. J., \& MacDorman, M. F. (2006) Infant mortality statistics from the 2003 period linked birth/infant death data set (National vital statistics reports; Vol. 54 No. 16). Hyattsville, MD: National Center for Health Statistics. (DHHS Publication No. PHS 2006-1120)

Reijneveld, S. A., DeKleine, M.J.K., VanBaar, A. L., Kollee, L. A. A., Verhaak, C. M., Verhulst, F. C., et al. (2006). Behavioural and emotional problems in very preterm and very low birthweight infants at age 5 years. Archives of Disease in Childhood-Fetal \& Neonatal Edition, 91, F423-F428.

Short, E. J., Klein, N. K., Lewis, B. A., Fulton, S., Eisengart, S., Kercsmar, C., et al. (2003) Cognitive and academic consequences of bronchopulmonary dysplasia and very low birth weight: 8-year-old outcomes. Pediatrics, 112, e359-e366.

Stucki, G., Stier-Jarmer, M., Grill, E., \& Melvin, J. (2005). Rationale and principles of early rehabilitation care after an acute injury or illness. Disability and Rehabilitation, 27, 353359

Wilson-Costello, D., Friedman, H. Minich, N., Fanaroff, A. A., \& Hack, M. (2005). Improved survival rates with increased neurodevelopmental disability for extremely low birth weight infants in the 1990's. Pediatrics, 115, 997-1003 
Morbidity and Mortality of Very Low Birth Weight Infant Graduates of a Level Three Neonatal Intensive Care Unit

Table 1

Demographic Data of Infants Surviving after Discharge

\begin{tabular}{lc}
\hline Characteristic & Percentage \\
\hline & Ethnicity \\
Hispanic & 69.3 \\
Black & 7 \\
White & 6 \\
Asian & 2.4 \\
Vietnamese & 1.6 \\
Arab & $<1$ \\
Filipino & $<1$ \\
Other/Unknown & 12 \\
\hline & \\
& \\
Female & 51 \\
Male & 49 \\
\hline Note. Ethnic categories are self-reported.
\end{tabular}


Morbidity and Mortality of Very Low Birth Weight Infant Graduates of a Level Three Neonatal Intensive Care Unit

Table 2

Distribution of Neurodevelopmental Results by Chronological Age

Chronological age in months as of last chart entry

Findings

$$
\begin{array}{rrrrrrr}
0-6 & 6.1-12 & 12.1-18 & 18.1-24 & 24.1-30 & >30 & \text { Total } \\
\mathrm{n}=10 & \mathrm{n}=34^{a} & \mathrm{n}=28 & \mathrm{n}=20^{a} & \mathrm{n}=20^{a} & \mathrm{n}=7 & \mathrm{n}=119^{a}
\end{array}
$$

\begin{tabular}{lccccccc}
\hline Abnormal Neuroevaluation & 4 & 19 & 10 & 12 & 12 & 7 & 64 \\
Speech/Language disorder & 0 & 1 & 1 & 4 & 3 & 7 & 16 \\
Behavior/Learning disorder & 0 & 0 & 0 & 1 & 1 & 0 & 2 \\
\hline
\end{tabular}

${ }^{a}$ excludes infants who have not received a complete neurological assessment. 
Morbidity and Mortality of Very Low Birth Weight Infant Graduates of a Level Three Neonatal Intensive Care Unit

Table 3

Distribution of Infant Morbidity by Chronological Age

\begin{tabular}{|c|c|c|c|c|c|c|c|}
\hline \multirow{3}{*}{ Morbidities } & \multicolumn{6}{|c|}{ Chronological age in months as of last chart entry } & \multirow{3}{*}{$\begin{array}{l}\text { Total } \\
\mathrm{n}=127\end{array}$} \\
\hline & $0-6$ & $6.1-12$ & $12.1-18$ & $18.1-24$ & $24.1-30$ & $>30$ & \\
\hline & $\mathrm{n}=10$ & $\mathrm{n}=39$ & $\mathrm{n}=28$ & $\mathrm{n}=21$ & $n=22$ & $\mathrm{n}=7$ & \\
\hline Chronic Lung disease & 2 & 10 & 7 & 5 & 6 & 3 & 33 \\
\hline Cerebral Palsy & 0 & 0 & 1 & 0 & 1 & 0 & 2 \\
\hline Hearing loss & 0 & 2 & 0 & 1 & 3 & 1 & 7 \\
\hline Visual loss & 0 & 0 & 0 & 1 & 0 & 0 & 1 \\
\hline Mental retardation & 0 & 0 & 0 & 0 & 0 & 1 & 1 \\
\hline Seizure disorder & 0 & 1 & 0 & 1 & 1 & 1 & 4 \\
\hline
\end{tabular}


Morbidity and Mortality of Very Low Birth Weight Infant Graduates of a Level Three Neonatal Intensive Care Unit

Table 4

Distribution of Infants with Hospital Readmissions ( $n=127)$

\begin{tabular}{cc} 
Hospital readmission & $\begin{array}{c}\text { Infants } \\
\text { No. (\%) }\end{array}$ \\
\hline 1 & $27(21.0 \%)$ \\
2 & $7(5.5 \%)$ \\
3 & $6(4.7 \%)$ \\
4 & $2(1.5 \%)$ \\
5 & $2(1.5 \%)$ \\
6 & $1(<1 \%)$
\end{tabular}

Note. Forty-five children (35\%) were readmitted to the hospital after discharge accounting for $\mathbf{7 8}$ hospital readmissions. 